\title{
Looking for an effective and non-invasive diagnostic test for endometriosis: where are we?
}

\author{
Pietro G. Signorile ${ }^{1}$, Alfonso Baldi ${ }^{1,2}$ \\ ${ }^{1}$ Fondazione Italiana Endometriosi, Rome, Italy; ${ }^{2}$ Department of Environmental, Biological and Pharmaceutical Sciences and Technologies, \\ Campania University "L. Vanvitelli", Caserta, Italy \\ Correspondence to: Alfonso Baldi. Fondazione Italiana Endometriosi, Rome, Italy. Email: alfonsobaldi@tiscali.it. \\ Provenance: This is an invited Editorial commissioned by Section Editor Hengwei Liu, MD, PhD (Department of Obstetrics and Gynecology, Union \\ Hospital, Tongji Medical College, Huazhong University of Science and Technology, Wuhan, China). \\ Comment on: Bockaj M, Fung B, Tsoulis M, et al. A method for electrochemical detection of brain derived neurotrophic factor (BDNF) in plasma. \\ Anal Chem 2018;90:8561-6.
}

Submitted Nov 05, 2018. Accepted for publication Nov 21, 2018.

doi: $10.21037 /$ atm.2018.11.46

View this article at: http://dx.doi.org/10.21037/atm.2018.11.46

Endometriosis is a very frequent estrogen-dependent gynecological disease, that affects up to $10 \%$ of all reproductive-aged women. The hallmark of endometriosis is the pathological growth of ectopic endometrial-like tissue outside of the uterine cavity (1). The prevalence, dramatically rises to $30-50 \%$ in women with chronic pelvic pain and infertility (2). The ectopic endometrial-like tissue can be found primarily on the pelvic peritoneum and ovaries, but it can grow also under the peritoneal surface. This last particular condition, named deep-penetrating endometriosis, is strongly associated with pelvic pain symptoms (3). Endometriosis patients have also a clinical history of infertility, and commonly experience pain also during menstruation and intercourse. Due to this incapacitating array of symptoms and problems caused by endometriosis, affected patients very often require extensive medical and surgical treatments with significant associated costs and risks (4). Because of this significant morbidity, endometriosis has relevant socio-economic implications with deleterious effect upon women's social functioning, personal life, and relationships with medical practitioners (4).

Despite the fact that this disease is very common and has a great social impact, to this date the pathogenesis, as well as the diagnosis and therapy are still not perfectly outlined. Retrograde menstruation and coelomic metaplasia are the most recognized pathogenetic hypotheses (5). In recent years, research conducted by our group and others have produced experimental evidence in favor of an alteration of the fine-tuning of the female genital system development during the fetal life as the pathogenetic event predisposing to the progression of endometriosis later in life (6-12).

The inevitable consequence of this lack of knowledge about the disease, is the fact that, still today, endometriosis is a significantly under-diagnosed and under-treated disease, with an unacceptably long estimated time interval between the onset of the symptoms and definitive diagnosis of $8-12$ years (1). This is largely due to the fact that most of the symptoms are non-specific and, to date, all the proposed non-invasive diagnostic approaches, such as blood tests, ultrasound and magnetic resonance imaging do not have sufficient specificity to allow a definitive diagnosis of endometriosis (13). At present, the conclusive diagnosis of endometriosis can be reached only by histological examination of the ectopic tissue growths collected following invasive surgical or exploratory procedures (1). Thus, an early and definitive diagnostic test of endometriosis without requiring invasive procedures is considered one of the priorities in gynecology. Such a test would permit to recognize endometriosis patients within a much narrower window of time after the onset of the symptoms, thus avoiding an extensive number of unnecessary invasive diagnostic and therapeutic procedures and years of unresolved, incapacitating pain. Such a test would also allow to reach the diagnosis of endometriosis in a group of women with sub-fertility. This would permit 
the identification of patients that could take advantage from laparoscopic surgery with earlier reduction of disease burden, improvement of fertility, and prevention of progression of the disease (14).

Numerous studies have been carried out with the aim of identifying endometriosis diagnostic markers in the serum. Unfortunately, none of the proposed biomarkers has been found to date, by themselves, to reach a clinically significant diagnostic specificity, as recently reviewed (15-17). As a consequence, none of the investigated biomarkers is presently used in routine clinical care.

An ideal diagnostic test for endometriosis should have high sensitivity and high specificity, displaying a low number of false negative and false positive results, so that no patients with actual endometriosis would be missed and no women without endometriosis would be selected for potentially unnecessary additional procedures. Our research group has been deeply involved in the identification of potential diagnostic circulating biomarkers of endometriosis. We used a proteomic 2D-gel analysisbased approach to identify proteins differentially expressed between a population of patients with endometriosis and a cohort of healthy women. This analysis allowed the definition of at least three potential diagnostic markers for endometriosis: zinc-alpha-2-glycoprotein, albumin, and complement C3 (18-20). ROC curve analyses confirmed the diagnostic potential of these markers in a robust cohort of endometriosis patients, with slightly different results for the three markers. Interestingly, the differential expression of these markers was independent from the cycle phase and from the endometriosis stage of the patients enrolled, thus minimizing any hormonal-related confounding and little to no stage specificity, indicating that these biomarkers are present early in the disease and remain unchanged in later stages. Moreover, the diagnostic potential of the three proteins was not completely overlapping, whereby each patient was individuated by at least one biomarker. Undeniably, the patients not identified by one of the markers were selected by the other two. This allows us to hypothesize that the combined use of the three markers could reach a diagnostic specificity superior to that of the markers taken individually (18-20).

In line with this research sits a recent article by Bockaj et al. (21). In this interesting manuscript, the authors identify the Brain Derived Neurotrophic Factor (BDNF) as a possible peripheral diagnostic marker for endometriosis. In addition, the authors define an original electrochemical detection technique for the recognition of BDNF from small amounts of peripheral blood, such as those obtained from a finger prick. This innovative method is based on a polymer-based chip with a nanoporous and a wrinkled gold film that acts as an electrode/sensing layer, thus permitting the electrochemical detection of even very small amount of BDNF in a small volume of plasma (21). The authors show that the chip operates in the range needed for the diagnosis of endometriosis $(200-2,000 \mathrm{pg} / \mathrm{mL})$ and that this sensitivity is similar to the one reached by conventional ELISA-based methods (21). This method combines two very important characteristics that a diagnostic test for endometriosis should have: high sensitivity and the ability to use very small amounts of blood collectable even by the patient herself, without the need for venipuncture. The authors propose to use this original screening method in combination with clinical and imaging data for the early detection of endometriosis.

Major limitations of this study are the small sample size, and the possibility that BDNF expression could be a sensitive but not sufficiently specific diagnostic marker for endometriosis. To note, variations in BDNF expression have been reported in several completely unrelated pathological conditions $(22,23)$. Nevertheless, the data are promising. Further studies on BDNF, as well as on other potential diagnostic markers, such as the ones identified by our research group (18-20), are indispensable to better define the real clinical impact of these assays on the diagnosis and management of endometriosis and to propose them to the scientific community has effective diagnostic tools for endometriosis.

It is to be hoped that research in the coming years will generate sufficient data to identify patients with endometriosis more precisely and at the earliest possible stage of the disease. We propose that it will be the combination of the data obtained from the circulating biomarkers and from imaging studies that will most likely represent in a not too distant future the most sensitive and effective combination of diagnostic approaches to endometriosis. The observations generated by our research group about the value of gadolinium-labelled anti-Mullerian hormone as a contrast agent for the diagnosis of stromal endometriosis lesions support this hypothesis $(24,25)$.

\section{Acknowledgements}

This work was supported by Fondazione Italiana Endometriosi. 


\section{Footnote}

Conflicts of Interest: PG Signorile and A Baldi are authors of a patent application (WO 2013/171655) related to the themes of the article.

\section{References}

1. Bulun SE. Endometriosis. N Engl J Med 2009;360:268-79.

2. Signorile PG, Campioni M, Vincenzi B, et al. Rectovaginal septum endometriosis: an immunohistochemical analysis of 62 cases. In Vivo 2009;23:459-64.

3. Baldi A, Campioni M, Signorile PG. Endometriosis: pathogenesis, diagnosis, therapy and association with cancer. Oncol Rep 2008;19:843-6.

4. Fuldeore M, Chwalisz K, Marx S, et al. Surgical procedures and their cost estimates among women with newly diagnosed endometriosis: a US database study. J Med Econ 2011;14:115-23.

5. Benagiano G, Brosens I. History of adenomyosis. Best Pract Res Clin Obstet Gynaecol 2006;20:449-63.

6. Signorile PG, Baldi F, Bussani R, et al. Ectopic endometrium in human fetuses is a common event and sustains the theory of mullerianosis in the pathogenesis of endometriosis, a disease that predisposes to cancer. J Exp Clin Cancer Res 2009;28:49.

7. Signorile PG, Baldi A. Endometriosis: new concepts in the pathogenesis. Int J Biochem Cell Biol 2010;42:778-80.

8. Signorile PG, Spugnini EP, Mita L, et al. Pre-natal exposure of mice to bisphenol A elicits an endometriosislike phenotype in female offspring. Gen Comp Endocrinol 2010;168:318-25.

9. Signorile PG, Baldi F, Bussani R, et al. New evidence of the presence of endometriosis in the human fetus. Reprod Biomed Online 2010;21:142-7.

10. Signorile PG, Baldi F, Bussani R, et al. Embryologic origin of endometriosis: analysis of 101 human female fetuses. J Cell Physiol 2012;227:1653-6.

11. Bouquet de Jolinière J, Ayoubi JM, Lesec G, et al. Identification of displaced endometrial glands and embryonic duct remnants in female fetal reproductive tract: possible pathogenetic role in endometriotic and pelvic neoplastic processes. Front Physiol 2012;3:444.

12. Crispi S, Piccolo MT, D'Avino A, et al. Transcriptional profiling of endometriosis tissues identifies genes related to organogenesis defects. J Cell Physiol 2013;228:1927-34.

13. Ballard K, Lowton K, Wright JT. What's the delay? A qualitative study of women's experience of reaching a diagnosis of endometriosis. Fertil Steril 2006;86:1296-301.

14. D'Hooghe TM, Mihalyi AM, Sisma P, et al. Why we need a noninvasive diagnostic test for minimal to mild endometriosis with high sensitivity. Gynecol Obstet Invest 2006;62:136-8.

15. May KE, Conduit-Hulbert SA, Villar J, et al. Peripheral biomarkers of endometriosis: a systematic review. Hum Reprod Update 2010;16:651-74.

16. Gupta D, Hull ML, Fraser I, et al. Endometrial biomarkers for the non-invasive diagnosis of endometriosis. Cochrane Database Syst Rev 2016;4:CD012165.

17. O DF, Flores I, Waelkens E, et al. Noninvasive diagnosis of endometriosis: Review of current peripheral blood and endometrial biomarkers. Best Pract Res Clin Obstet Gynaecol 2018;50:72-83.

18. Signorile PG, Baldi A. Serum biomarker for diagnosis of endometriosis. J Cell Physiol 2014;229:1731-5.

19. Signorile PG, Baldi A. Supporting evidences for potential biomarkers of endometriosis detected in peripheral blood. Data Brief 2015;5:971-4.

20. Signorile PG, Baldi A. Prototype of Multiplex Bead Assay for Quantification of Three Serum Biomarkers for In Vitro Diagnosis of Endometriosis. J Cell Physiol 2016;231:2622-7.

21. Bockaj M, Fung B, Tsoulis M, et al. A method for electrochemical detection of brain derived neurotrophic factor (BDNF) in plasma. Anal Chem 2018;90:8561-6.

22. Polyakova M, Stuke K, Schuemberg K, et al. BDNF as a biomarker for successful treatment of mood disorders: a systematic and quantitative meta-analysis. J Affect Disord 2015;174:432-40.

23. Smeele P, d'Almeida SM, Meiller C, et al. Brainderived neurotrophic factor, a new soluble biomarker for malignant pleural mesothelioma involved in angiogenesis. Mol Cancer 2018;17:148.

24. Signorile PG, Baldi A. A tissue specific magnetic resonance contrast agent, Gd-AMH, for diagnosis of stromal endometriosis lesions: a phase I study. J Cell Physiol 2015;230:1270-5.

25. Signorile PG, Baldi A. New evidence in endometriosis. Int J Biochem Cell Biol 2015;60:19-22.

Cite this article as: Signorile PG, Baldi A. Looking for an effective and non-invasive diagnostic test for endometriosis: where are we? Ann Transl Med 2018;6(Suppl 2):S106. doi: 10.21037/atm.2018.11.46 